\title{
Investigation of Shock Waves in Non-Ideal Gas under the Action of Magnetic Field
}

\author{
Kanti Pandey, Praveen Prakash Pathak \\ Department of Mathematics \& Astronomy, Lucknow University, Lucknow, India \\ Email: pandey_kanti@yahoo.co.in
}

How to cite this paper: Pandey, K. and Pathak, P.P. (2017) Investigation of Shock Waves in Non-Ideal Gas under the Action of Magnetic Field. Advances in Pure Mathematics, 7, 583-596.

https://doi.org/10.4236/apm.2017.710035

Received: September 30, 2017

Accepted: October 28, 2017

Published: October 31, 2017

Copyright $\odot 2017$ by authors and Scientific Research Publishing Inc. This work is licensed under the Creative Commons Attribution International License (CC BY 4.0).

http://creativecommons.org/licenses/by/4.0/

\begin{abstract}
In present paper, certain aspect of shock wave in non-ideal gas, when magnetic field is orthogonal to the trajectories of the gas particles and electrical conductivity is taken to be infinite, is investigated. Considering one-dimensional unsteady non-planer motion, basic equations, its general solution and formation of shock-wave, conservation laws and jumps conditions, variation of area of non-uniform cross section and analytical solution of strong non planer shock is obtained.
\end{abstract}

\section{Keywords}

Shock-Waves, Non-Ideal Gases, Magnetic Field

\section{Introduction}

The assumption that the medium is an ideal gas is no more valid when the flow takes place in extreme conditions. Anisinov and Spiner [1] have studied a problem of point explosion in low density non ideal gas by taking the equation of state in a simplified form which describes the behaviour of medium satisfactorily. Robert and $\mathrm{Wu}$ [2] have studied the gas that obeys a simplified Vander Waal's equation of state. Assuming that the piston is moving with time according to law given by Steiner and Hirschler [3], Vishwakarma et al. [4] have investigated the one dimensional unsteady self-similar flow behind a strong shock, driven out by a cylindrical or spherical piston in a medium which is assumed to be non-ideal and which obey the simplified Vander-Waal's equation of state as considered by Robert and Wu [5]. Madhumita and Sharma [6] have considered the model equation for a low density gas, which describes the behaviour of the medium satisfactorily for implosion problems where the temperature attained by the gas motion in the strong shock limit is very high. Vishwakarma et al. [7] 
have considered the propagation of magneto-gas dynamic cylindrical and spherical shock waves in gas with decreasing density.

At higher temperature the gas is in ionised state, i.e. the gas is electrically conducting. The study of flow problems of such gases has been receiving considerable interest for the last several decades [8]-[20]. Such studies are important in connection with astrophysical [21] [22] and geophysical [23] problems also. Re-entry problem of intercontinental ballistic missiles and supersonic projectiles are two engineering applications of such flows. Because of such engineering applications many engineers and aerodynamicists joining the astrophysicists and geophysicists have extensively studied the dynamics of electrically conducting gases moving in a magnetic field. When an electrically conducting gas moves in magnetic field, electric field is induced in it and electric currents start flowing in the gas. The magnetic field exerts forces on these currents which may considerably modify the flow. Conversely, the currents themselves modify the magnetic field. Thus we have a very complex interaction between the magnetic and gas dynamic phenomena and hence the gas flow must be explained by combining the field equations with those of gas dynamic equations.

In present paper, certain aspect of shock wave in non-ideal gas, when magnetic field is orthogonal to the trajectories of the gas particles and electrical conductivity is taken to be infinite, is investigated. Considering one-dimensional unsteady non-planer motion, basic equations, its general solution and formation of shock-wave, conservation laws and jumps conditions, variation of area of non-uniform cross section and analytical solution of strong non planer shock is obtained. It is concluded that the manner of formation of a shock wave in magnetogasdynamics is the same as that in ordinary gasdynamics. But since $a_{e}$ is larger than a speed of sound for ideal gas case, the shock wave formation will be faster in magneto-gas-dynamics for non-ideal gas.

\section{Basic Equations and Formation of Shock Wave}

If we consider the case when electrical conductivity is very high and transverse magnetic field is to be orthogonal to the trajectories of fluid particles, then equations governing the one dimensional motion of unsteady non-ideal gas in presence of magnetic field when dissipative effects are neglected are given by [11]

$$
\begin{gathered}
\rho_{, t}+u \rho_{, x}+\rho u_{, x}=0 \\
u_{, t}+u u_{, x}+\frac{1}{\rho} p_{, x}+\frac{\mu H}{\rho} H_{, x}=0 \\
H_{, t}+u H_{, x}+H u_{, x}=0,
\end{gathered}
$$

and

$$
E_{, t}+\left\{u\left(E+p_{t}\right)\right\}_{, x}=0,
$$

where $\rho, u, H, p_{t}$ are density, fluid velocity, magnetic field, total pressure defined by 


$$
p_{t}=p+\frac{\mu H^{2}}{2}
$$

$p$ being non-ideal gas pressure and $\frac{\mu H^{2}}{2}$ is magnetic pressure, $\mu$ being magnetic permeability and a comma followed by an index implies partial derivative with respect to that index. $E$ is the total energy density given by

$$
E=\rho e+\frac{1}{2} \rho u^{2},
$$

where $\mathrm{e}$ is the internal energy given by

$$
e=C_{v} T+\frac{\mu H^{2}}{2}=\frac{V-b}{\gamma-1} p+\frac{\mu H^{2}}{2},
$$

Tbeing temperature and $V=\frac{1}{\rho}$.

Following Robert and $\mathrm{Wu}$ [5], we consider the equation of state for non-ideal gas as

$$
p=\frac{\rho R T}{1-b \rho}=\frac{R T}{V-b},
$$

$R$ being universal gas constant, $b$ being the internal volume of the gas molecules, which is known in term of the molecular interaction potential in high temperature gases and $\gamma$ being ratio of specific heats.

To find the exact solution of energy Equation (2.4) such that flow is isentropic and density is a function of $\mathrm{u}$ alone i.e.

$$
\rho=f(u),
$$

we can write Equations (2.1) and (2.2) in following form

$$
u_{, t}+u u_{, x}=-\frac{f}{f^{\prime}} u_{, x}
$$

and

$$
u_{, t}+u u_{, x}=-\frac{a_{e}^{2}}{f} f u_{, x}
$$

respectively, where

$$
a_{e}^{2}=\left\{\frac{\gamma p}{\rho(1-b \rho)}+\frac{\mu H^{2}}{\rho}\right\}
$$

is effective speed of sound and is a function of $\rho$ alone in the present case.

$$
\frac{\gamma p}{\rho(1-b \rho)} \text { is speed of sound for non-ideal gas and } c^{2}=\frac{\mu H^{2}}{\rho} \text { is Alfven ve- }
$$
locity.

From Equations (2.7) and (2.8) and taking into consideration that

$$
f^{\prime}=f_{, u}=\rho_{, u}
$$

we have 


$$
\frac{f^{\prime}}{f}=\frac{1}{\rho} \frac{\partial \rho}{\partial u}= \pm \frac{1}{a_{e}}
$$

Integrating above equation we have

$$
u= \pm \int_{\rho_{0}}^{\rho} \frac{a_{e}(\rho)}{\rho} \mathrm{d} \rho .
$$

Substuting Equation (2.9) into Equation (2.7) and after certain manipulation we have

$$
u_{, t}+\left(u \pm a_{e}\right) u_{, x}=0 \text { and } \rho_{, t}+\left(u \pm a_{e}\right) \rho_{, x}=0 .
$$

The general solution of above equations is

$$
u=F_{1}\left[x-\left(u \pm a_{e}\right) t\right], \rho=F_{2}\left[x-\left(u \pm a_{e}\right) t\right],
$$

where $F_{1}$ and $F_{2}$ are arbitrary constants.

Let us consider the solution

$$
u=F_{1}\left[x-\left(u+a_{e}\right) t\right] .
$$

This solution means that the variation of $\mathrm{u}$ with respect to a point moving with velocity $\left(u+a_{e}\right)$ is zero, i.e. the disturbance is propagated with an instantaneous velocity $\left(u+a_{e}\right)$. If the velocity $\mathrm{u}$ much smaller than $a_{e}$, we have $u=F_{1}\left[x-a_{e} t\right]$. Thus $a_{e}$ is the velocity of propagation of an infinitesimal disturbance in the present case. Hence we call $a_{e}$ the effective speed of sound. For wave of finite amplitude, the velocity of propagation is different at different points in the flow field, and the shape of the wave will be distorted as the wave propagates. For wave of the type of Equation (2.12), the velocity of propagation at the crest is larger than that at the trough. Hence the crest will overtake the trough. As a result, a shock will form and the assumption of isentropic flow breaks down. Qualitatively, the manner of formation of a shock wave in magnetogasdynamics is the same as that in ordinary gasdynamics. But since $a_{e}$ is larger than a speed of sound for ideal gas case, the shock wave formation will be faster in magneto-gasdynamics for non-ideal gas. The solution $u=F_{1}\left[x-\left(u-a_{e}\right) t\right]$ represents an expansion wave while $F_{1}\left[x-\left(u+a_{e}\right) t\right]$ represents a compression wave. The expansion wave will smooth itself out as the wave propagates while the compression wave will form a shock as the wave propagates.

As systems of fundamental Equations (2.1) to (2.3) are of hyperbolic type, we may use the well known method of characteristics to solve such problems. There are four sets of characteristic curves for this case given as:

Double roots of $x_{, t}=u$ as flow lines

$$
\text { and } x_{t}=u \pm a_{e} \text {, characteristic lines }
$$

Along the flow lines $x_{, t}=u$, we have

$$
\frac{H}{\rho}=\text { constant }
$$

and 


$$
S=\text { constant }
$$

Along the characteristic line $x_{, t}=u \pm a_{e}$, we have respectively

$$
\mu_{e} H\left[H_{, t}+\left(u \pm a_{e}\right) H_{, x}\right] \pm \rho a_{e}\left[u_{, t}+\left(u \pm u_{e}\right) u_{, x}\right]+\left[p_{, t}+\left(u \pm a_{e}\right) p_{, x}\right]=0
$$

\section{Conservation Laws and Jump Conditions}

Conservation equation in this case can be written as

$$
\begin{gathered}
\rho_{1}=\rho_{0} \beta \\
H_{1}=H_{0} \beta \\
\rho_{1}\left(u_{0}-u_{1}\right)=\rho_{0} u_{0} \\
p_{1}+\frac{1}{2} \mu H_{1}^{2}+\rho_{1}\left(u_{0}-u_{1}\right)^{2}=p_{0}+\frac{1}{2} \mu H_{0}^{2}+\rho_{0} u_{0}^{2} \\
\frac{1}{2}\left(u_{0}-u_{1}\right)^{2}+\frac{p_{1}}{(\gamma-1) \rho_{1}\left(1+b \rho_{1}\right)}+\frac{p_{1}}{\rho_{1}}+\frac{\mu H_{1}^{2}}{\rho_{1}} \\
=\frac{1}{2} u_{0}^{2}+\frac{p_{0}}{(\gamma-1) \rho_{0}\left(1+b \rho_{0}\right)}+\frac{p_{0}}{\rho_{0}}+\frac{\mu H_{0}^{2}}{\rho_{0}}
\end{gathered}
$$

where

$$
\begin{gathered}
u_{1}=\left\{\frac { 2 \beta } { 2 + ( 1 + \overline { b } \beta ) ( \gamma - 1 ) ( 1 - \beta ) } \left\{\frac{a_{0}^{2}}{\gamma(1+\bar{b})}(1+\bar{b}(\beta+1)\right.\right. \\
\left.\left.+(1+\bar{b})(\gamma-1)(1+\bar{b} \beta))+\frac{b_{0}^{2}}{2}(\beta+1+(1+\bar{b} \beta)(\gamma-1)(1-\beta))\right\}\right\} \\
p_{1}=p_{0}+\frac{2 \rho_{1}(\beta-1)}{2+(1+\bar{b} \beta)(\gamma-1)(1+\beta)}\left\{\frac{a_{0}^{2}}{\gamma(1+\bar{b})}(1+\bar{b}(\beta+1)\right. \\
\left.+(1+\bar{b})(\gamma-1)(1+\bar{b} \beta))+\frac{b_{0}^{2}}{4}\left((1-\beta)^{2}(1+\bar{b} \beta)(\gamma-1)\right)\right\} \\
u_{0}=\frac{\beta}{\beta-1} u_{1}
\end{gathered}
$$

$p_{0}$ is the constant ambient pressure, subscript ' 1 ' and ' 0 ' refer to state just behind and just ahead of the shock and $\beta$ is given by following equation

$$
\begin{aligned}
& (1-\gamma) \bar{b} \frac{M^{2}}{M_{A}^{2}} \beta^{3}+\beta^{2}\left[\frac{M^{2}}{M_{A}^{2}}\{2+(\gamma-1) \bar{b}-\gamma\}+\frac{2}{\gamma(1+\bar{b})}\{\gamma \bar{b}\}+(\gamma-1) \bar{b} M^{2}\right] \\
& +\beta\left[(\gamma-1) M^{2}+\gamma \frac{M^{2}}{M_{A}^{2}}+2-(\gamma-1) \bar{b} M^{2}\right]-(\gamma+1) M^{2}=0
\end{aligned}
$$

where $M^{2}=\frac{a_{0}^{2}}{u_{0}^{2}}, M_{A}^{2}=\frac{c_{0}^{2}}{u_{0}^{2}}$ and $\bar{b}=b \rho_{0}$ whose square and higher power are neglected.

The strong shock condition in present case is given as: 


$$
\begin{gathered}
u_{1}=\frac{2 u(1-\alpha)}{\gamma+1}, \quad p_{1}=\frac{2 u^{2} \rho_{0}(1-\alpha)}{\gamma+1}, \quad \rho_{1}=\rho_{0} \frac{\gamma+1}{(\gamma-1)+2 \alpha}, \\
a_{1}=\frac{u}{\gamma+1}\{2 \gamma(1-b \rho)\}^{1 / 2}\{(\gamma-1)+2 b \rho\}^{1 / 2}
\end{gathered}
$$

\section{Characteristics Rules}

In this section we consider the method of characteristic as described by Whitham [24] to find change in area of a non uniform tube in case of pure magneto-gasdynamic non-ideal conducting gas. If we consider one dimensional formulation for flow in a tube of a given cross-sectional area, where

$A(x)=A_{0}=$ constant , in $x<0$, and the shock is initially moving in this section with constant Mach number $M_{0}$. We consider that the shock is to be produced by a piston moving with appropriate constant speed far back in the uniform section. The piston is still providing the thrust to keep the shock moving, but there are no changes due to this and the changes are entirely due to the cross-sectional area. Though the flow is not strictly one dimensional but if the cross-section $A(x)$ does not vary too rapidly, the equations obtained by averaging across the tube will provide a good approximation of one dimensional flow. Thus for this case the equations governing the motion reduces into following form

$$
\begin{gathered}
\rho_{, t}+u \rho_{, x}+\rho u_{, x}+\rho u \frac{A_{, x}(x)}{A(x)}=0 \\
u_{, t}+u u_{, x}+\frac{1}{\rho} p_{, x}+\frac{\mu H}{\rho} H_{, x}=0 \\
H_{, t}+u H_{, x}+H u_{, x}+H u \frac{A_{, x}(x)}{A}=0
\end{gathered}
$$

and

$$
\rho T\left(S_{, t}+u S_{, x}\right)=\frac{J^{2}}{\sigma}
$$

where $S, J, \sigma$, are entropy, current density vector and electrical conductivity respectively. In case of infinite electrical conductivity $\sigma \rightarrow \infty$, hence above equation reduces to

$$
\rho T\left(S_{, t}+u S_{, x}\right)=0
$$

case corresponding to isentropic flow. Above system of equation may be combined to give following differential equation along positive characteristics:

$$
\begin{gathered}
\frac{\mathrm{d} x}{\mathrm{~d} t}=u+a_{e} \\
\mathrm{~d} p+\mu H \mathrm{~d} H+\rho a_{e} \mathrm{~d} u+\frac{\rho a_{e}^{2} u}{u+a_{e}} \frac{\mathrm{d} A}{A}=0
\end{gathered}
$$

Since in this case we are dealing with pure magneto-gasdynamics shock wave in non-ideal gas, using the values of flow variable just behind the shock, given by 
Equations (3.1), (3.2),(3.6),(3.7) and (3.8), into the characteristic Equation (4.7), after certain simplifications, we have following differential equation which will provide change of cross-sectional area of the tube

$$
\frac{\mathrm{d} A}{A}=F(\beta) \mathrm{d} \beta
$$

where

$$
\begin{aligned}
& F(\beta)=-\left\{\frac{R(\beta)+\beta H_{0} \mathrm{~d} H_{0}+\rho_{0} \beta N(\beta) \frac{\mathrm{d} L(\beta)}{\mathrm{d} \beta}}{\left\{\beta \rho_{0} N^{2}(\beta) L(\beta)\right\} /\{L(\beta)+N(\beta)\}}\right\} \\
& R(\beta)=\frac{1}{2+(1+\bar{b} \beta)(\gamma-1)(1+\beta)}\left[\frac{2(\beta-1) p_{0} \bar{b}}{1+\bar{b}}\{1+(1+\bar{b})(\gamma-1)\}\right. \\
& +\frac{h^{2}(1-\beta)}{2}\{(\gamma-1)(1-\bar{b} \beta)-\bar{b}(\beta-1)(\gamma-1)\} \\
& +\left\langle\frac{p_{0}}{1+\bar{b}}\{1+\bar{b}(\beta+1)+(1+\bar{b})(\gamma-1)(1+\bar{b} \beta)\}+\frac{h^{2}}{4}\left\{(1-\beta)^{2}(\gamma-1)(1-\bar{b} \beta)\right\}\right\rangle \\
& \left.*\left\langle\frac{2+2(1+\bar{b} \beta)(\gamma-1)(1+\beta)-2(\beta-1)\{(1+\beta)(\gamma-1) \bar{b}+(1+\bar{b} \beta)(\gamma-1)\}}{2+(1+\bar{b} \beta)(\gamma-1)(1+\beta)}\right\rangle\right] \\
& L(\beta)=\left\{\frac { 2 \beta } { 2 + ( 1 + \overline { b } \beta ) ( \gamma - 1 ) ( 1 + \beta ) } \left[\frac{a_{0}^{2}}{\gamma(1+\bar{b})}\{1+\bar{b}(1+\beta)+(1+\bar{b})(\gamma-1)(1+\bar{b} \beta)\}\right.\right. \\
& \left.\left.+\frac{b_{0}^{2}}{2}\{(1+\beta)+(1+\bar{b} \beta)(\gamma-1)(1-\beta)\}\right]\right\}^{1 / 2} \\
& N(\beta)=\sqrt{\frac{\gamma p_{0}(\beta)}{\rho_{0}(1-\alpha)}+\frac{\mu H_{0}^{2}}{\rho_{0}}} \\
& \frac{\mathrm{d} L}{\mathrm{~d} \beta}=\frac{1}{L(\beta)\{2+(1+\bar{b} \beta)(\gamma-1)(1-\beta)\}} \\
& *\left[\left\langle\frac{a_{0}^{2} \beta \bar{b}}{\gamma(1+\bar{b})}\{1+(1+\bar{b})(\gamma-1)\}+\frac{c_{0}^{2} \beta}{2}\{1-(1+\bar{b} \beta)(\gamma-1)+(\gamma-1)(1-\beta) \bar{b}\}\right\rangle\right. \\
& +\left\langle\frac{a_{0}^{2}}{\gamma(1+\bar{b})}\{1+\bar{b}(\beta+1)+(1+\bar{b})(\gamma-1)(1+\bar{b} \beta)\}\right. \\
& \left.+\frac{c_{0}^{2}}{2}\{(1+\beta)+(1+\bar{b} \beta)(\gamma-1)(1-\beta)\}\right) \\
& \left.*\left\langle\frac{\{2+(1+\bar{b} \beta)(\gamma-1)(1-\beta)\}-\beta\{\bar{b}(\gamma-1)(1-\beta)-(1+\bar{b} \beta)(\gamma-1)\}}{2+(1+\bar{b} \beta)(\gamma-1)(1-\beta)}\right\rangle\right]
\end{aligned}
$$




$$
\begin{aligned}
p_{1}= & p_{0}+\frac{2(\beta-1)}{2+(1+\bar{b} \beta)(\gamma-1)(1+\beta)}\left[\frac{p_{0}}{1+\bar{b}}\{1+\bar{b}(\beta+1)\right. \\
& \left.+(1+\bar{b})(\gamma-1)(1+\bar{b} \beta)\}+\frac{\mu H^{2}}{4}\left\{(1-\beta)^{2}(\gamma-1)(1-\bar{b} \beta)\right\}\right]
\end{aligned}
$$

\section{Analytical Solution for Non-Planer Wave}

In this section we shall deal with analytical solution for strong non-planer shock in non-ideal magneto-gasdynamics. Assuming the electrical conductivity to be infinite and the direction of the magnetic field orthogonal to the trajectories of the gas particle, the governing equation for the one dimensional unsteady non planer motion can be written as [17]

$$
\begin{gathered}
\rho_{, t}+u \rho_{, x}+\rho u_{, x}+\frac{(m-1) \rho u}{x}=0 \\
u_{, t}+u u_{, x}+\rho^{-1}\left(p_{, x}+h_{, x}\right)=0 \\
p_{, t}+u p_{, x}-a^{2}\left(\rho_{, t}+u \rho_{, x}\right)=0 \\
h_{, t}+u h_{, x}+2 h u_{, x}+2 h u \frac{m-1}{x}=0
\end{gathered}
$$

where $u$ is the gas velocity, $\rho$ is the density, $p$ is the pressure, $\gamma$ is the constant specific heat ratio, $t$ is the time, $x$ is the single spatial coordinate being either axial in flows with planer geometry, or radial in cylindrically symmetric flows, $a^{2}=\frac{\gamma p}{\rho(1-b \rho)}$ is the equilibrium speed of sound, $h$ is the magnetic pressure defined by $h=\mu H^{2} / 2$ with $\mu$ as magnetic permeability $H$ is a transverse magnetic field, $\mathrm{m}=1$ and 2 correspond, respectively, to planer and cylindrical symmetry. A comma followed by a subscript " $x$ " or " $t$ " denotes the partial differentiation unless stated otherwise. The system of Equation (5.1) to (5.4) is supplemented with an equation of state $p=\frac{\rho R T}{1-b \rho}$, where $R$ is gas constant and $T$ is the temperature. It is well known that of shock wave may be initiated in the flow region and once it is formed, it will propagate by separating the portion of continuous region. At shock, the correct generalized solution satisfied the Rankine-Hugoniot jump condition. Let $x=\chi(t)$ be the strong shock with the shock speed $u_{0}=\mathrm{d} \chi / \mathrm{d} t$ propagating into the medium characterized by,

$$
\rho=\rho_{0}(x), u=0, p=p_{0}(x), h=h_{0}(x)
$$

Therefore the boundary conditions at the shock front in present case can be written as

$$
\begin{gathered}
\rho=\frac{\rho_{0}(\gamma+1)}{\gamma-1}\left[1-\frac{2 \alpha}{\gamma-1}-\frac{2}{\gamma-1}\left(\frac{a_{0}}{u_{0}}\right)^{2}\right], \\
u=\frac{2}{\gamma+1} u_{0}\left[(1-\alpha)-\left(\frac{a_{0}}{u_{0}}\right)^{2}\right],
\end{gathered}
$$




$$
\begin{gathered}
p=\frac{2 \rho_{0} u_{0}^{2}}{\gamma+1}\left\{(1-\alpha)-\frac{\gamma-1}{2 \gamma}\left(\frac{a_{0}}{u_{0}}\right)^{2}\right\}-\frac{1}{2} C_{0} \rho_{0} u_{0}^{2}\left(\frac{\gamma+1}{\gamma-1}\right)^{2}\left\{1-\frac{4 \alpha}{\gamma-1}-\frac{4}{\gamma-1}\left(\frac{a_{0}}{u_{0}}\right)^{2}\right\}, \\
h=\frac{1}{2} C_{0} \rho_{0} u_{0}^{2}\left(\frac{\gamma+1}{\gamma-1}\right)^{2}\left\{1-\frac{4 \alpha}{\gamma-1}-\frac{4}{\gamma-1}\left(\frac{a_{0}}{u_{0}}\right)^{2}\right\},
\end{gathered}
$$

where $a_{0}$ is the sound speed of the undisturbed medium, $C_{0}=2 h_{0} / \rho_{0} u_{0}^{2}$ is the shock Cowling number and the suffix " $o$ " denote evaluation of the flow parameters just ahead of the shock respectively.

If we assume that an explosion takes place over a plane or along a line accompanied by release of a finite amount of energy $E$, a plane or cylindrical strong shock is instantaneously formed which begins to propagate outward into the medium at rest. Thus the total energy $E$ inside a blast wave is equal to the energy supplied by the explosive and thus constant. Thus total energy is given by

$$
E=4 \pi \int_{0}^{u_{0}}\left(\frac{1}{2} \rho u^{2}+\frac{p}{(\gamma-1)(1-b \rho)}+h\right) x^{m-1} \mathrm{~d} x,
$$

which represents the sum of the kinetic and internal energy. Since the initial energy input $E_{0}$ of explosion is very large, the shocks speed $u_{0} \gg a_{0}$ so that $a_{0} / u_{0} \rightarrow 0$ in the strong shock limit. Therefore the Rankine-Hugoniot jump conditions in the strong shock waves can be written as

$$
\begin{aligned}
& \rho=\frac{\rho_{0}(\gamma+1)}{\gamma-1}\left[1-\frac{2 \alpha}{\gamma-1}\right], u=\frac{2}{\gamma+1} u_{0}(1-\alpha) \\
& p=\frac{2 \rho_{0} u_{0}^{2}}{\gamma+1}(1-\alpha)-\frac{1}{2} C_{0} \rho_{0} u_{0}^{2}\left(\frac{\gamma+1}{\gamma-1}\right)^{2}\left\{1-\frac{4 \alpha}{\gamma-1}\right\}, \\
& h=\frac{1}{2} C_{0} \rho_{0} u_{0}^{2}\left(\frac{\gamma+1}{\gamma-1}\right)^{2}\left\{1-\frac{4 \alpha}{\gamma-1}\right\}
\end{aligned}
$$

With the help of Equation (4.5.11), Equation (4.5.12) can be written as

$$
\begin{aligned}
& p=\frac{\gamma-1}{2} \rho u^{2}\left\{1+\frac{2}{\gamma-1}\right\}-\frac{1}{8} C_{0} \rho u^{2}\left(\frac{\gamma+1}{\gamma-1}\right)^{2}\left(\frac{\gamma+1}{1-2 \alpha}\right)\left\{1+\frac{2 \alpha}{\gamma-1}\right\}, \\
& h=\frac{1}{8} C_{0} \rho u^{2}\left(\frac{\gamma+1}{\gamma-1}\right)^{2}\left(\frac{\gamma+1}{1-2 \alpha}\right)\left\{1+\frac{2 \alpha}{\gamma-1}\right\}
\end{aligned}
$$

After using Equation (5.13), the governing Equations (5.1), (5.2) and (5.4) can be transformed to

$$
\begin{gathered}
\frac{\partial u}{\partial t}+u \frac{\partial u}{\partial x}+\frac{1}{2 \rho}(\gamma-1)\left(1+\frac{2 \alpha}{\gamma-1}\right)\left\{u^{2} \frac{\partial \rho}{\partial x}+2 u \frac{\partial u}{\partial x}\right\}=0 \\
\frac{\partial u}{\partial t}+u \frac{\partial u}{\partial x}+\frac{1}{2}(\gamma-1) u\left(1+\frac{2 \alpha}{\gamma-1}\right)\left\{\frac{\partial u}{\partial x}+\frac{(m-1) u}{x}\right\}=0 \\
\frac{\partial u}{\partial t}+u \frac{\partial u}{\partial x}+\frac{1}{2} u\left\{\frac{\partial u}{\partial x}+\frac{(m-1) u}{x}\right\}=0
\end{gathered}
$$


Using Equations (5.14) and (5.15), and then integrating with respect to ' $x$ ', we get

$$
\rho u x^{-(m-1)}=\xi(t)
$$

where $\xi(t)$ is an arbitrary function of integration.

Using the solution (5.17), Equation (5.1) reduced to

$$
\frac{\partial u}{\partial t}-2(m-1) \frac{u^{2}}{x}-\frac{u}{\xi} \frac{\mathrm{d} \xi}{\mathrm{d} t}=0
$$

On solving Equations (5.15) and (5.18), we have

$$
u=-\eta \frac{x}{\xi} \frac{\mathrm{d} \xi}{\mathrm{d} t}
$$

where

$$
\eta=\frac{2}{\gamma+1}\left\{1+\frac{2 \alpha m}{\gamma+1}+\frac{(m-1)(\gamma+3)}{\gamma+1}\right\}^{-1}
$$

Putting value of " $u$ " from Equation (5.19) in Equation (5.18) and then integrating, we obtained

$$
\xi=\frac{\xi_{0}}{t^{\lambda}}
$$

where

$$
\lambda=\left\{1+\frac{2 \alpha m}{\gamma+1}+\frac{(m-1)(\gamma+3)}{\gamma+1}\right\}\left\{1+\frac{2 \alpha m}{\gamma+1}+\frac{(m-1)(\gamma-1)}{\gamma+1}\right\}^{-1}
$$

and $\xi_{0}$ is arbitrary constant.

With the help of boundary condition (5.11), we can obtain the analytical expression of the distance $\chi$ as

$$
\chi=t^{\frac{\gamma+1}{2(1-\alpha) w}}
$$

where

$$
w=\frac{(\gamma+1)+(m-1)(\gamma-1)+2 \alpha m}{2} .
$$

Consequently, with the help of Equation (5.19), the analytical solutions of the flow variables and total energy in the presence of the magnetic field is given by

$$
\begin{gathered}
u=x w^{-1} t^{-1} \\
\rho=\xi_{0} w x^{m-2} t^{1-\lambda} \\
p=\left[\frac{\gamma-1}{2}\left(1+\frac{2 \alpha}{\gamma-1}\right)-\left\{\frac{C_{0}}{8} \frac{(\gamma+1)^{3}}{(\gamma-1)(1-2 \alpha)}\left(1+\frac{2 \alpha}{\gamma-1}\right)\right\}\right] \xi_{0} x^{m} w^{-1} t^{-(1+\lambda)} \\
h=\left\{\frac{C_{0}}{8} \frac{(\gamma+1)^{3}}{(\gamma-1)(1-2 \alpha)}\left(1+\frac{2 \alpha}{\gamma-1}\right)\right\} \xi_{0} x^{m} w^{-1} t^{-(1+\lambda)}
\end{gathered}
$$

In view of the above solution (5.22), the analytical expression for the total 
energy is given by

$$
\begin{aligned}
E= & \frac{2 \pi}{m w} \xi_{0}\left[\left\{\frac{2(\gamma-1)+\alpha(2-\gamma)}{2(\gamma-1)(1-\alpha)}\right\}+\frac{C_{0}}{8} \frac{(\gamma+1)^{3}}{(\gamma-1)^{2}(1-2 \alpha)}\right. \\
& \left.\times\left(1+\frac{2 \alpha}{\gamma-1}\right)\left\{\frac{\gamma(1-\alpha)+(\alpha-2)}{(\gamma-1)(1-\alpha)}\right\}\right]
\end{aligned}
$$

\section{Result and Discussion}

In this paper, an attempt is made to discuss certain aspect of shock wave in non-ideal gas when magnetic field is orthogonal to the trajectories of the gas particles and electrical conductivity is taken to be infinite. It is concluded that due to presence of magnetic field shock wave being formed quickly. Variation of pressure, velocity density, and area change is investigated for different values of magnetic field and internal volume of gas molecules. The analytical solution for non-planer strong shock wave is also obtained. For two different values of internal volume of gas molecules $(\alpha=0,0.025,0.05)$ variation of velocity, density, gas pressure and magnetic pressure is analysed through graphs. Figure 1 shows the variation of velocity for $(\alpha=0,0.025,0.05)$ and it is concluded that as $\alpha$ is increasing velocity is increasing and always less than velocity of ideal gas. Figure 2 shows the variation of density for $(\alpha=0,0.025,0.05)$ and it is concluded that as $\alpha$ is increasing density is decreasing and always less than density of ideal gas. Figure 3 shows the variation of magnetic pressure for $(\alpha=0,0.025,0.05)$ and it is concluded that as $\alpha$ is increasing magnetic pressure has an increasing tendency but is always greater than the ideal gas case. Figure 4(a) and Figure 4(b) show variation for pressure for different Cowling number $\left(C_{0}=0.02,0.05\right)$ and different values of internal volume of gas molecules $(\alpha=0,0.025,0.05)$. It is concluded that pressure has increasing tendency and is always greater than ideal gas pressure.

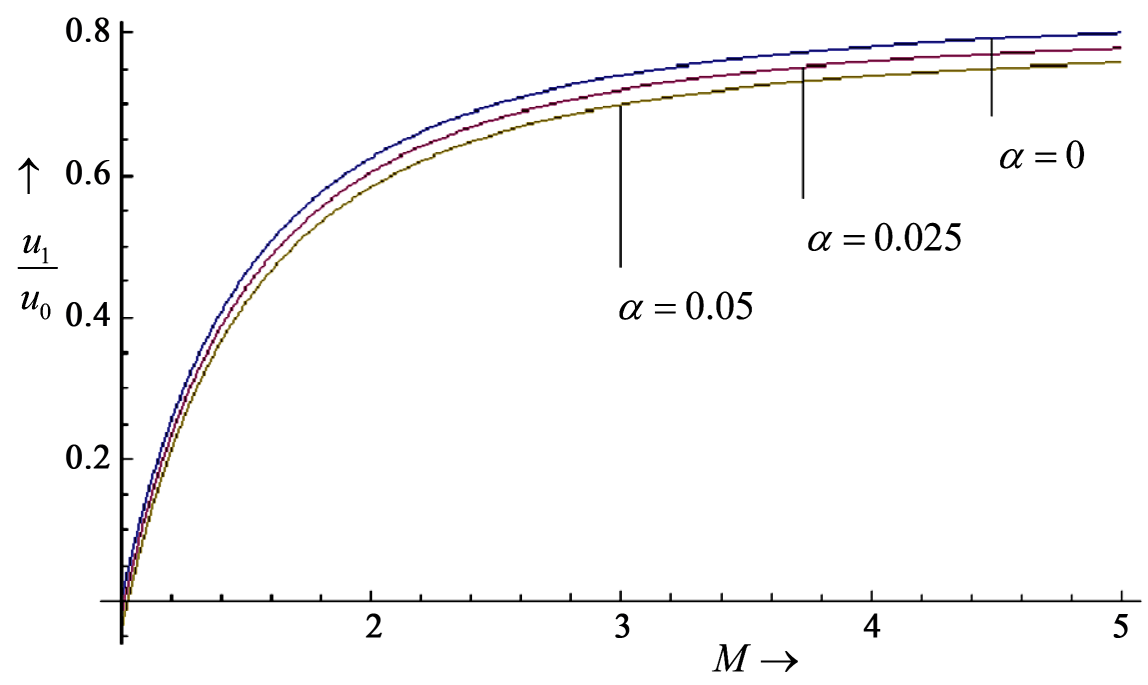

Figure 1. Variation of velocity " $u_{1} / u_{0}$ " for different values of the Mach number " $M$ ". 


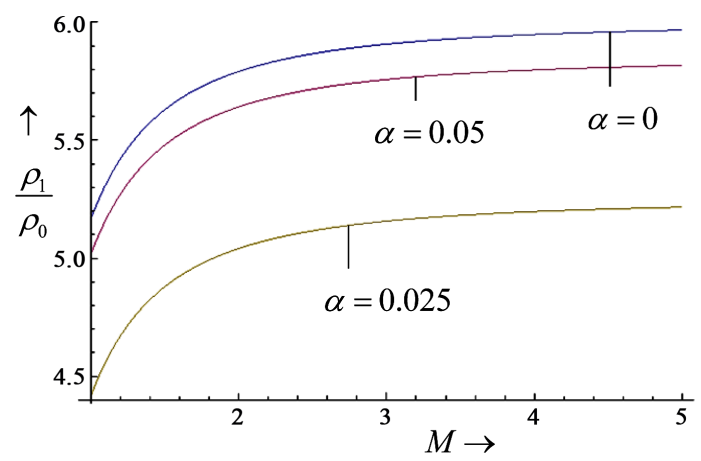

Figure 2. Variation of density " $\rho_{1} / \rho_{0}$ ” for different values of Mach number " $M$ ".

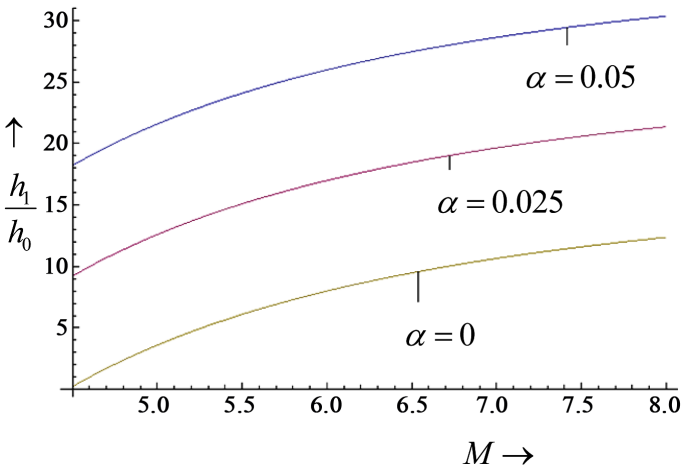

Figure 3. Variation of magnetic field " $h_{1} / h_{0}$ ” for different values of Mach number "M".

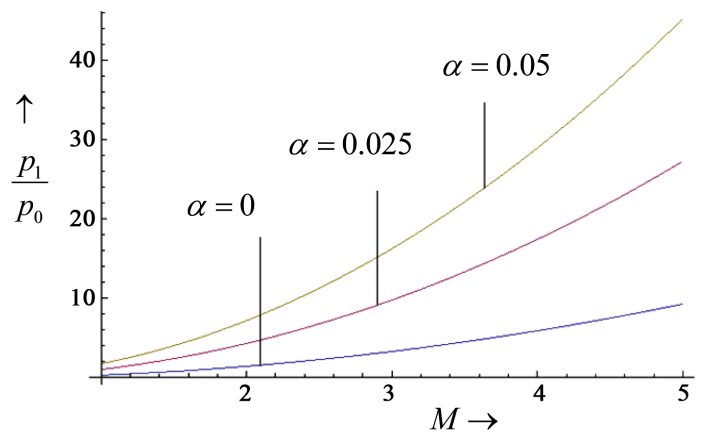

(a)

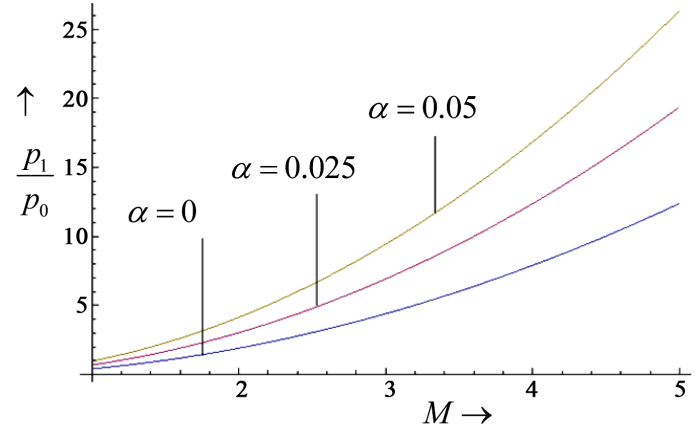

(b)

Figure 4. (a) Variation of pressure " $p_{1} / p_{0}$ " for different values of the Mach number " $\mathrm{M}$ " at cowling number $C_{0}=0.5$; (b) Variation of pressure " $p_{1} / p_{0}$ " for different values of Mach number "M" at cowling number $C_{0}=0.2$. 


\section{Acknowledgements}

One of the authors, Kanti Pandey, is grateful to UGC for providing financial assistance in the preparation of this article.

\section{References}

[1] Anisinov, S.I. and Spiner, O.M. (1972) Motion of an Almost Ideal Gas in the Presence of a Strong Point Explosion. Journal of Applied Mathematics and Mechanics, 36, 883-887. https://doi.org/10.1016/0021-8928(72)90144-x

[2] Robert, P.H. and Wu, C.C. (1993) Shock-Wave Propagation in a Sonoluminescing Gas Bubble. Physical Review Letters, 70, 3424-3427.

https://doi.org/10.1103/physrevlett.70.3424

[3] Steiner, H. and Hirschler, T. (2002) A Self Similar Solution of a Shock Propagation in Dusty Gas. European Journal of Mechanics-B/ Fluids, 21, 371-380. https://doi.org/10.1016/S0997-7546(02)01181-0

[4] Vishwakarma J.P., Chaube, V. and Patel, A. (2007) Self-Similar Solution of Shock Propagation in a Non Ideal Gases. International Journal of Applied Mechanics and Engineering, 12, 813-829.

[5] Robert, P.H. and Wu, C.C. (2003). The Shock-Wave Theory in Sonoluminescence. In: Srivastava, R.C., et al., Eds., Shock Focusing Effect in Medical Science and Sonoluminescence, Springer Verlag, Berlin, 1-27.

[6] Madhumita, G. and Sharma, V.D. (2004) Imploding Cylindrical and Spherical Shock Waves in a Non Ideal Medium. Journal of Hyperbolic Differential Equations, 1, 521-530. https://doi.org/10.1142/S0219891604000184

[7] Vishwakarma, J.P. and Yadav, A.K. (2002) Propagation of Magneto-Gasdynamic Cylindrical and Spherical Shock Waves in Gas with Decreasing Density. Indian Journal of Theoretical Physics, 50, 125-135.

[8] Kanwal, R.P. (1960) Flow behind Shock Waves in Conducting Gases. Proceedings of the Royal Society A, 25, 263-268. https://doi.org/10.1098/rspa.1960.0148

[9] Mitchener, M. and Vinokur, V. (1963) Radiation Smoothing of Shocks with and without a Magnetic Field. Physics of Fluids, 6, 1682-1692.

[10] Nariboli, G.A. and Secrest, B.G. (1967) Weak Discontinuities in Magneto Gasdynamics in the Presence of Dissipative Mechanism. Tensor N.S., 18, 22-25.

[11] Pai, S.I. (1962) Magnetogasdynamics and Plasma Dynamics. Springer Verlag, Berlin.

[12] Pai, S.I. (1963) Some Considerations of Radiation Magneto Gasdynamics. Proceedings of the Symposium on Nonlinear Problems, University of Wisconsin Press, Madison, 47-67.

[13] Pandey, K. and Mishra, R.S. (1981) Unsteady Flows behind Two Dimensional Curved Magnetogasdynamics Wave in Radiating Gases. Progress in Mathematics, 15, 27-37.

[14] Pant, J.C. (1965) Some Coincidences in Magneto Fluid Dynamics. Tensor (N.S.), 16, 269-279.

[15] Pant, J.C. (1968) Oblique Regular Reflection of a Plane Shock in Presence of a Transeverse Magnetic Field. ZAMM, 48, 73-85. https://doi.org/10.1002/zamm.19680480202

[16] Pant, J.C. and Mishra, R.S. (1968) Existence and Uniqueness of Flows behind Three Dimensional Unsteady and Pseudo-Stationary Curved Shock Waves in Conducting Fluids. Journal of the Indian Mathematical Society, 32, 39-54. 
[17] Ram, R. (1981) Analytical Solution of the Problem of Violent Explosions in a Plasma of Varying Density. Acta Mechanica, 40, 75-86. https://doi.org/10.1007/BF01170691

[18] Rosenau, P. and Frankenthal, S. (1976) Equatoril Propagation of Axisymmetric Magnetohydrodynamic Shocks. Physics of Fluids, 19, 1989-1999.

https://doi.org/10.1063/1.861424

[19] Vishwakarma, J.P., Srivastava, R.C. and Kumar, A. (1987) Magneto-Gas-Dynamics for the Flows behind a Spherical Shock Wave. Astrophysics and Space Science, 129, 45-52. https://doi.org/10.1007/BF00717856

[20] Verma, B.G. (1982) Strong Magnetogasdynamic Shock Waves with Increasing Energy. Indian Journal of Pure and Applied Mathematics, 13, 981-988.

[21] Burgers, J.M. and Van de Hulst, H.C. (1949) Problems of Cosmic Aerodynamics. Central Air Documents Office, Dayton.

[22] Burgers, J.M. and Van de Hulst, H.C. (1955) Gasdynamics of Cosmic Clouds. Interscience Publishers Inc., New York.

[23] Elsasser, W.M. (1956) Hydromagnetic Dynamo Theory. Reviews of Modern Physics, 28, 135-145. https://doi.org/10.1103/RevModPhys.28.135

[24] Whitham, G.B. (1974) Linear and Non-Linear Waves. John Wiley \& Sons, New York. 